\title{
Das Schweizer Gesundheitswesen ist ein System
}

Andreas Walter

Korrespondenz:

Andreas Walter

Siemens Schweiz AG

Leiter Sector Healthcare

Freilagerstrasse 40

CH-8047 Zürich

andreas.a.walter@siemens.com

\section{Das Gesundheitswesen Schweiz ist ein System}

Das Schweizer Gesundheitswesen folgt keinem starren Regelwerk. Vielmehr ist es ein komplexes System, das durch Interaktionen zwischen Ursache und Wirkung geprägt ist. Dabei versucht jeder Akteur, die gesetzten Rahmenbedingungen zu seinem Vorteil zu nutzen. Was sich zuerst nach Wettbewerb anhört, löst in der Praxis ein kostentreibendes und akteurzentriertes System aus. Denn der Wettbewerb entsteht durch die aktuellen Anreize nicht wie gewünscht unter den Leistungserbringern und Kostenträgern selbst, sondern mehrheitlich in einem Abgrenzungsprozess zwischen Leistungserbringern und Versicherern, Versicherern und Kantonen sowie Kantonen und den interkantonalen Versorgungsplanungen. Dazu kommt, dass der Kanton aktuell als Gesetzgeber, Auftraggeber, Betreiber, Leistungseinkäufer, Kostenträger und Schlichtungsstelle gleich mehreren Zielkonflikten ausgesetzt ist.

Dieses komplexe adaptive System lässt keine Nutzung des Gesamtpotentials zu. Damit wird der Patient immer weiter an den Rand gedrängt,

\section{Handbuch «Gesundheitswesen Schweiz im Umbruch»}

Das Schweizer Gesundheitswesen befindet sich in einem grundlegenden Umbruch. Hinter den einschneidenden Veränderungen stehen als Hauptkräfte der stetig wachsende Wettbewerb unter den Akteuren, neue Finanzierungs- und Vertragsmodelle zwischen Leistungserbringern und Leistungsfinanzierern sowie der demographische und epidemiologische Wandel in unserer Gesellschaft. Gleichzeitig erhöhen Anpassungen der gesetzlichen Rahmenbedingungen den Druck auf die Akteure. Die Spitäler, alle Leistungserbringer, Krankenversicherer und die Industrie müssen ihre Strategie und die Leistungsprozesse in den nächsten 2-5 Jahren auf diese Veränderungskräfte ausrichten.

Diese Thesen liegen dem eben erschienenen Handbuch «Gesundheitswesen Schweiz im Umbruch» zugrunde, in dem sich 40 Experten aus Forschung, Wissenschaft und Praxis in 50 Fachbeiträgen vertieft mit den Charakteristika des Schweizer Gesundheitswesens befassen und den was zu einer Verzerrung des Wertschöpfungsprozesses im System führt.

Das Ergebnis dieser Anreize hat nachhaltig ein akteurzentriertes Gesundheitssystem gefördert, dessen Auswirkungen heute flächendeckend spürbar sind: heterogene System- und Finanzierungslandschaften, ineffiziente Abläufe zwischen den Akteuren sowie ein stetig steigender Organisations- und Regulationsaufwand, um das System handlungsfähig und finanzierbar zu halten (Abb. 1). Der ausgelöste Trend im Schweizer Gesundheitswesen ist ein kontinuierlicher Anstieg der Komplexität. Dadurch werden das aktuelle System und die Rollen der Akteure, wie es Willy Oggier in seinem Artikel «Regulatoren unter Druck» [1] beschreibt, in Zukunft in Frage gestellt.

\section{Rationales Verhalten}

In der Systemtheorie wird zwischen zwei zentralen Entscheidungsmustern unterschieden. Die individuelle Rationalität beschreibt die Entscheidungsfindung, bei der der Nutzen aus einer Kooperation mindestens so hoch sein muss wie der Nutzen, den der Akteur aus eigener Kraft

Veränderungsprozess der nächsten Jahre detailliert beschreiben. Dabei stehen folgende Fragen im Zentrum: Was lässt das Schweizer Gesundheitswesen in Zukunft komplexer werden? Welche Prozessschritte sind durch die Komplexitätssteigerung betroffen und werden zu Vitalprozessen? Mit welchen Massnahmen kann die Vitalität entwickelt werden, um der Komplexitätssteigerung voraus zu sein?

Als Herausgeber des Handbuchs zeichnen mit Willy Oggier, Andreas Walter, Serge Reichlin und Michael Egli vier Persönlichkeiten, die in unterschiedlichsten Bereichen über eine langjährige Berufserfahrung im Gesundheitswesen verfügen. Für die SÄZ haben verschiedene Autoren einen Artikel zu einem zentralen Thema des Handbuchs verfasst. Wir präsentieren unserer Leserschaft diese Beiträge in den Nummern 15 bis 18-19/2008 als Kurzserie.

Weitere Informationen zum Handbuch «Gesundheitswesen Schweiz im Umbruch» unter www. ehealthcare.ch 
erreichen kann. Die Summe der individuellen rationalen Entscheidungen wird dadurch im Sinne eines gemeinsamen übergeordneten Ziels irrational. Dieses Paradoxon wurde eingehend von Alexrod mit dem Ansatz des Gefangenendilemmas untersucht [2].

Die kollektive Rationalität steht für eine rationale Lösung eines Problems, die die Summe der Einzelnutzen aller vorhandenen Akteure maximiert. Mit der kollektiven Rationalität wird somit das gesamte Potential eines Systems zum

\section{Abbildung 1}

Aktuelle Anreize fördern ein akteurzentriertes Gesundheitssystem.

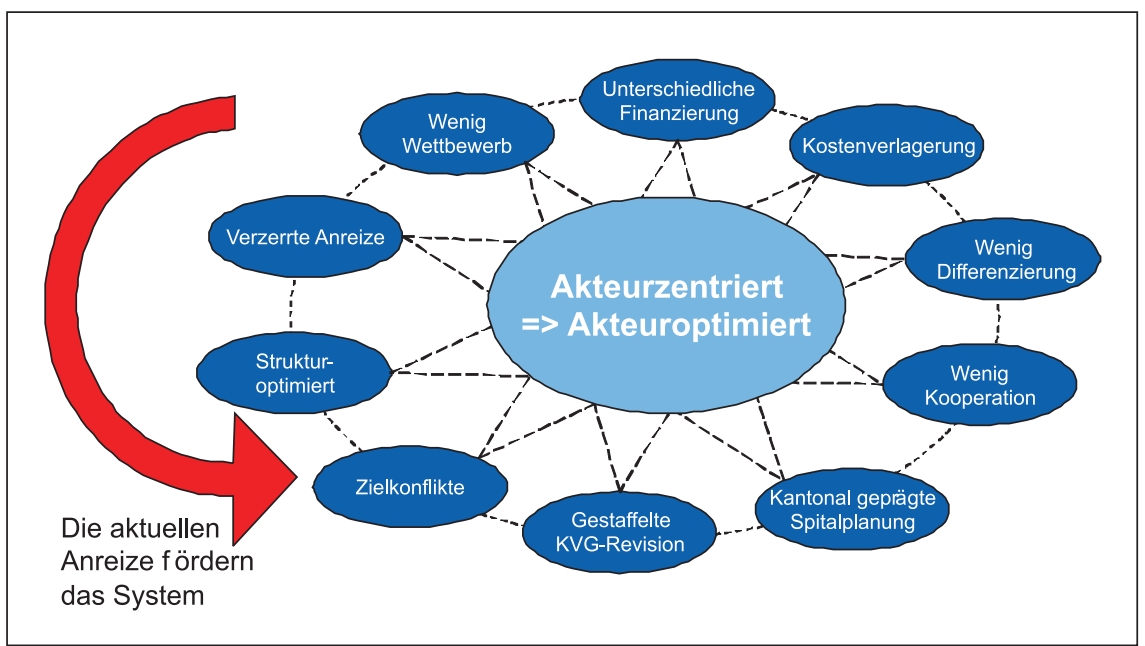

maximalen Nutzen jeden einzelnen Akteurs entwickelt. Die kollektive Rationalität ist in der Systemtheorie ein Sonderfall, der sich in seiner Umsetzbarkeit reziprok zur Komplexität verhält. Das heisst, je komplexer ein System wird, desto weniger werden die Akteure darin kollektiv rational entscheiden. Eines der wichtigsten Voraussetzungen für einen kollektiv rationalen Entscheid sind verbindliche Abmachungen und hohes Vertrauen unter den Akteuren [3].

Im Schweizer Gesundheitswesen, das durch eine hohe Komplexität als Folge individueller rationaler Entscheidungen geprägt ist, wird der Ruf nach zentraler Kontrolle und Vereinfachung laut. Das Problem, das sich aus Sicht der Systemtheorie dabei ergibt, ist, dass die Lösungen für eine neue Ordnung ebenfalls komplex sind. In der Definition von Lebensfähigkeit von Vester [4] wird deutlich, dass die Reduktion der Komplexität durch eine Reduktion der Entscheidungsfreiheit der Akteure keine nachhaltige Lebensfähigkeit entstehen lässt. Ein zentralistisch hierarchisch geführtes System und verliert dabei wesentliche Kriterien für einen nachhaltigen Umgang mit Ressourcen und ein qualitatives
Wachstum. Die Frage, die sich daraus stellt, ist, wie Komplexität konstruktiv genutzt und wie ein adaptives System trotz hoher Eigendynamik und Negativschlaufen in eine nachhaltige Richtung entwickelt werden kann.

Ein Modell, das dieses Spannungsfeld zwischen Lebenskraft und Komplexität beschreibt, wird im folgenden Kapitel aufgezeigt.

\section{Vitalität in Relation zur Komplexität?}

Die Lebenskraft (Vitalität) eines Akteurs steht relativ zur Komplexität seiner Umwelt. Steigt die Komplexität der Umwelt, sinkt die Vitalität des Akteurs. Ist der Akteur nicht mehr fähig, die Komplexität seiner Umwelt $\mathrm{zu}$ beherrschen, bricht seine Vitalität ein. Ein Vitalitätsverlust zeigt sich häufig durch zunehmend reaktives Handeln. Der Akteur wirkt chaotisch und wenig zielgerichtet. Die bekannten Verhaltensmuster und Werkzeuge werden immer weniger wirksam und der Ruf nach neuen Strategien immer lauter (Abb. 2).

Damit wird die Vitalität eines Akteurs nachhaltig von der Komplexität seiner Umwelt beeinflusst. Dieser Beeinflussung stellt sich der Akteur mit seiner Kompetenz und dem Freiheitsgrad seiner Entscheidungen, seine Vitalität nutzbringend einzusetzen.

Als wesentliche Bausteine der Lebenskraft beschreibt Vester [4] die Selbstorganisation und die Kooperation. Feedbackschlaufen sind zentrale Bestandteile dieses Mechanismus, die auch die Fähigkeit der Adaption in komplexen Systemen ermöglichen. Selbstorganisation und Kooperation als Konzepte haben einen auf Prozesse ausgerichteten Charakter, der nicht Strukturen, sondern Abläufe zur Förderung der Lebensfähigkeit nutzt. Durch Verbindung und Austausch gelingt es den Partnern, einen vitalen Vorsprung gegenüber nicht kooperierenden Akteuren zu erzielen.

Auf das Schweizer Gesundheitswesen angewendet, zeigt sich hier die grosse Herausforderung der regulativen Eingriffe, die die Vitalität der einzelnen Akteure durch Entscheidungsfreiheit und Kooperation im Sinne des Gesamtsystems fördern sollen. Gleichzeitig wird dabei die Komplexität erhöht, was viele Akteure in ihrer Vitalität gefährdet.

\section{Fehlendes Gesamtkonzept für eine nachhaltige Lebensfähigkeit}

Eine aktive Systemgestaltung benötigt übergeordnete Anreize, welche die Akteure zu Systemeffizienz und -effektivität antreiben. Der in den letzten Jahren immer stärker wirkende Kostendruck und die gestaffelte KVG-Revisionen wurden innerhalb der aktuellen Struktur und akteur- 


\section{Abbildung 2}

Relation zwischen der Vitalität eines Akteurs und der Komplexität seiner Umwelt.

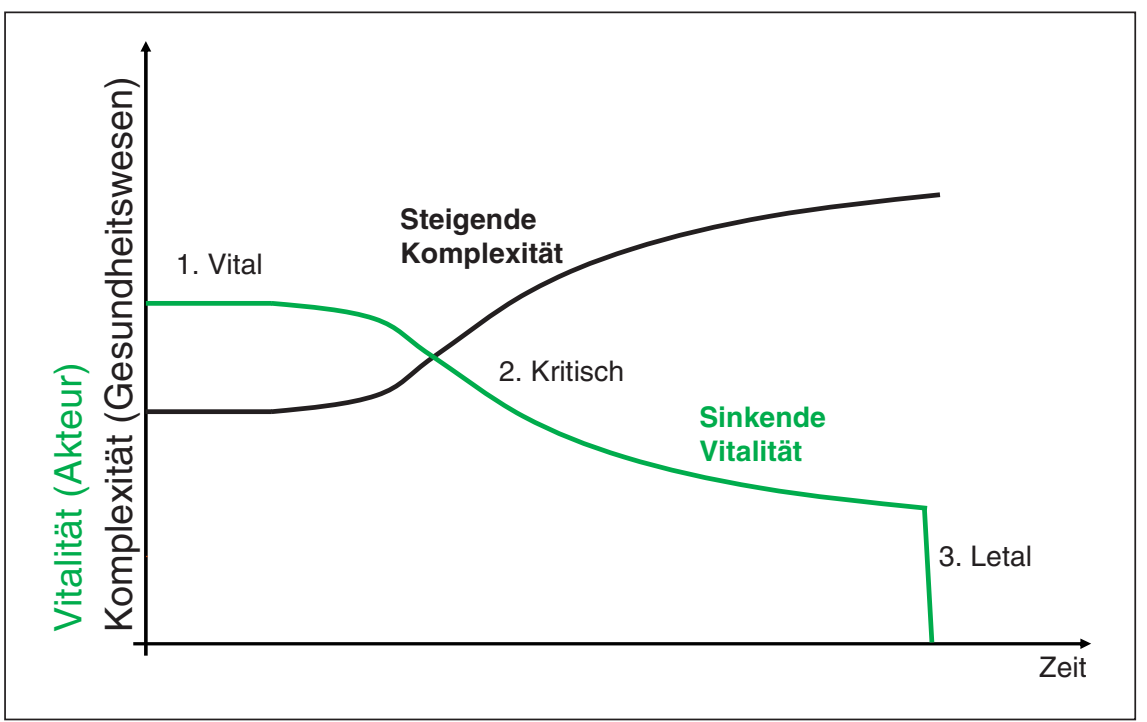

zentriert kompensiert. Organisationsstrukturen wurden gestrafft, Softwarewerkzeuge wurden evaluiert und implementiert, mit denen die bestehenden Strukturen nachgebaut wurden. Die Akteure handeln im aktuellen Gesundheitswesen noch vital und können die sich verändernden Rahmenbedingungen für sich immer wieder vitalisierend adaptieren. Diese individuell adaptierte Strukturoptimierung verhindert eine nachhaltige Reform für eine wirkungsvolle Förderung der Lebensfähigkeit. Aus der blockierten Situation heraus polarisiert das System immer mehr, was die Dominanz und die Handlungsmotive der Akteure immer weiter auseinandertreibt. In der ausgelösten Komplexitätssteigerung kommen die aktuellen Strategien und Strukturoptimierungen immer mehr an ihre Grenzen. Gleichzeitig verhindern sie aber auch
Gesamtkonzepte für die Förderung nachhaltiger Lebensfähigkeit. Da diese Entwicklung durch quantitative Grössen wie z.B. Mengenausweitung oder Kostenverlagerung angetrieben wird und das qualitative Wachstum durch fehlende Prozessoptimierung gehemmt wird, ist die Entwicklung endlich, und die Akteure werden im System an die Grenzen ihrer Handlungsfähigkeit stossen.

\section{Fazit}

Die Akteure des Schweizer Gesundheitswesens beherrschen die Komplexität des Systems. Die aktuellen Anreize lassen die Akteure individuell rational handeln, was einer kollektiven rationalen Gesamtentwicklung und Nutzenpotentialrealisierung im System entgegenwirkt. Aufgrund der komfortablen Ausgangslage sehen die Akteure keinen Grund, etwas zu ändern. Beide Verhaltensmuster lassen das Gesundheitswesen oberflächlich stabil wirken, in der tieferen Analyse ist es irrational. Die aktuellen Anreize steigern das quantitative Wachstum auf Kosten einzelner, was diesen Trend systemtheoretisch endlich werden lässt. Die sich stellenden Fragen sind, wie lange die Akteure die dabei entstehende Komplexität noch bewältigen können und wann ein Paradigmawechsel notwendig wird.

\section{Literatur}

1 Oggier W. Regulatoren unter Druck. Schweiz Ärztezeitung. 2008;89(15):639-42.

2 Axelrod R. Die Evolution der Kooperation. München: Oldenbourg; 2000,

3 Holland JH. Studying complex adaptive systems. J Syst Science Complexity. 2006;19(1):1-8.

4 Vester F. Die Kunst, vernetzt zu denken. München: dtv; 2002. 